\title{
The transcriptome of Utricularia vulgaris, a rootless plant with minimalist genome, reveals extreme alternative splicing and only moderate sequence similarity with Utricularia gibba
}

\author{
Jiř́ Bárta ${ }^{1}$, James D Stone ${ }^{2,3}$, Jiří Pech', Dagmara Sirová', Lubomír Adamec ${ }^{4}$, Matthew A Campbell ${ }^{5}$ \\ and Helena Štorchová ${ }^{*}$
}

\begin{abstract}
Background: The species of Utricularia attract attention not only owing to their carnivorous lifestyle, but also due to an elevated substitution rate and a dynamic evolution of genome size leading to its dramatic reduction. To better understand the evolutionary dynamics of genome size and content as well as the great physiological plasticity in this mostly aquatic carnivorous genus, we analyzed the transcriptome of Utricularia vulgaris, a temperate species with well characterized physiology and ecology. We compared its transcriptome, namely gene content and overall transcript profile, with a previously described transcriptome of Utricularia gibba, a congener possessing one of the smallest angiosperm genomes.
\end{abstract}

Results: We sequenced a normalized cDNA library prepared from total RNA extracted from shoots of U. vulgaris including leaves and traps, cultivated under sterile or outdoor conditions. 454 pyrosequencing resulted in more than 1,400,000 reads which were assembled into 41,407 isotigs in 19,522 isogroups. We observed high transcript variation in several isogroups explained by multiple loci and/or alternative splicing. The comparison of $U$. vulgaris and U. gibba transcriptomes revealed a similar distribution of GO categories among expressed genes, despite the differences in transcriptome preparation. We also found a strong correspondence in the presence or absence of root-associated genes between the U. vulgaris transcriptome and U. gibba genome, which indicated that the loss of some root-specific genes had occurred before the divergence of the two rootless species.

Conclusions: The species-rich genus Utricularia offers a unique opportunity to study adaptations related to the environment and carnivorous habit and also evolutionary processes responsible for considerable genome reduction. We show that a transcriptome may approximate the genome for gene content or gene duplication estimation. Our study is the first comparison of two global sequence data sets in Utricularia.

Keywords: Transcriptome, Root-associated genes, Alternative splicing, Utricularia vulgaris

\section{Background}

Members of the rootless genus Utricularia (Lentibulariaceae) are the most versatile and cosmopolitan among carnivorous plants, exhibiting great morphological and ecophysiological plasticity [1-3]. Approximately 50 species of Utricularia are aquatic or amphibious, growing in

\footnotetext{
* Correspondence: storchova@ueb.cas.cz

${ }^{2}$ Institute of Experimental Botany CAS, Rozvojová 263 6- Lysolaje, Praha 16502, Czech Republic

Full list of author information is available at the end of the article
}

standing, nutrient-poor humic waters. While their ecology and carnivorous habit have been researched previously [3], increasing attention has been given to the peculiarities of Utricularia genomes - miniature size in many species within the family [4,5], highly increased nucleotide substitution rates across the genomes of all three cellular compartments: mitochondrial, plastid, and nuclear [6-9], and to the extremely dynamic evolution of genome size at the level of species or even single populations $[4,10]$. Utricularia gibba possesses one of the smallest haploid 
angiosperm genomes known, approximately one-half that of Arabidopsis thaliana, with chromosomes of bacterial size [4,5]. U. gibba was the subject of the first broad survey of nuclear gene transcripts in Utricularia species $[11,12]$, revealing several interesting aspects of their physiology and morphology. Supporting physiological data, the global transcript analysis revealed specific expression patterns of genes involved in respiration, DNA repair, ROS detoxification, and nutrient uptake in different plant tissues. The sequencing and analysis of the $U$. gibba genome [13] additionally revealed a compressed genome architecture with highly reduced intergenic regions and nearly free of retrotransposons.

Utricularia spp. have since been identified as prime candidates for further research on the complexities of plant ecophysiology associated with carnivory, metagenomic surveys of trap microbial communities, novel plant nitrogen/nutrient utilization pathways, the ecology of prey attraction, whole-plant and trap comparative development, and the evolution of a minimalist angiosperm genome [3,5,14-20]. Utricularia gibba, however, is not a good candidate species for many ecological and physiological experiments due to its minute size and extremely small traps. We have therefore chosen the ecologically well-characterized temperate Utricularia vulgaris [3,16-18] as our model for a broad transcriptome analysis. Its ecophysiology is subtly but meaningfully distinct from that of $U$. gibba, offering the possibility for a comprehensive comparison of genome-wide expression patterns between the two species.

In this study, we report the results of 454 GS-FLX Titanium sequencing of a polyA-selected and normalized cDNA library from $U$. vulgaris, derived from a pooled sample of multiple tissue types, including functional annotation of expressed gene content. We compared this transcriptome to the $U$. gibba transcriptome [12] and showed that, despite different methods of preparation and tissue composition, the overall gene expression pattern and gene distribution among GO categories were very similar between the two species. We also analysed several cases of alternative splicing (AS) in the $U$. vulgaris transcriptome, including a gene for which this post-transcriptional process has not been investigated in any plant species.

Although any transcriptome should be viewed as incomplete, it may serve as an acceptable proxy for the genome in a species without complete genomic information, such as $U$. vulgaris, provided that it is prepared from multiple tissues and various environmental conditions [21,22]. We demonstrate the usefulness of the $U$. vulgaris transcriptome for the identification of gene losses and duplications during the course of evolution of the genus Utricularia.

\section{Results}

\section{Transcriptome assembly}

In total, $1,405,703$ reads were generated by 454 pyrosequencing of the $U$. vulgaris normalized cDNA library, $1,389,835$ of them passed built-in quality filtering. $91.5 \%$ of the initial, raw reads were assembled by Newbler 2.7 and produced 19,522 isogroups containing 41,407 isotigs, roughly corresponding to the individual transcripts. In addition, 64,188 singletons longer than 100 nt were obtained. Isotigs and singletons were combined together into a unique transcript (UT_U.vulgaris) data set representing the $U$. vulgaris transcriptome. To facilitate the comparison between our data and the $U$. gibba transcriptome published by [12], raw reads of $U$. gibba were downloaded from DNA Data Bank of Japan (DDBJ) under the submission SRA029151 and assembled by Newbler 2.7 using the same parameters as adopted for the $U$. vulgaris transcriptome (UT_U.gibba). Table 1 compares the transcriptome assemblies of the two species. Our $U$. vulgaris data set consisted of nearly twice as many raw reads, a higher proportion of which assembled into contigs, than the $U$. gibba dataset. The $U$. vulgaris assembly also resulted in a higher number of isogroups and much higher (about three fold) number of isotigs. Furthermore, our $U$. vulgaris assembly produced only 64,188 singletons compared to the 99,900 singletons remaining after de novo $U$. gibba assembly. The $U$. vulgaris data set contained about 2.1 isotigs per isogroup, whereas only 1.2 isotigs per isogroup, on average, were found in the $U$. gibba assembly. The much higher number of isotigs in the $U$. vulgaris transcriptome, both relative (per isogroup) and absolute, was at least partly caused by the method of cDNA library preparation. Our $U$. vulgaris cDNA library was normalized, increasing the number of rare transcripts represented by isotigs.

\section{Transcriptome annotation}

39,006 U. vulgaris isotigs (96\%) gave significant BLAST hit against the NCBI nr protein database (BLASTX algorithm, e-value cutoff $10^{-5}$ ). These sequences were further annotated using the BLAST2GO annotation pipeline. 30,392 isotigs (73\% of total isotigs) were successfully annotated. 9,794 isotigs (33\% of annotated isotigs) were assigned with enzyme codes (E.C.). The average level of annotations in GO hierarchy was 5,868 . The total number of assigned Gene Ontology terms was 212,122 (Table 2).

Of the total 58,363 U. vulgaris singletons, 23,212 (40\%) gave a significant BLAST hit against the NCBI nr protein database under the same parameters as used for isotigs. 14,536 singletons ( $25 \%$ of total singletons) were successfully annotated and 4,121 singletons (28\% of annotated singletons) were assigned with E.C. The average level of annotations in GO hierarchy was 5,791. The total number of assigned Gene Ontology terms was 90,735. The much lower proportion of $U$. vulgaris singletons yielding 
Table 1 Transcriptomes comparison

\begin{tabular}{|c|c|c|}
\hline & Utricularia vulgaris & Utricularia gibba \\
\hline Biological source of RNA & Shoots, cultivated under sterile conditions & Shoots and flowers natural conditions \\
\hline cDNA library preparation & Oligo dT enrichment normalized library & Oligo dT enrichment without normalization \\
\hline inputFile NumReads & 1405703 & 817792 \\
\hline numAlignedReads & $1271732 ; 91.5 \%$ of total & $707880 ; 86.56 \%$ of total \\
\hline Number of Assembled & 1151110 & 638520 \\
\hline Number of Partially assembled & 120448 & 69288 \\
\hline Number of Singletons & 64188 & 99900 \\
\hline Number Outlier (e.g. chimeras) & 23169 & 9967 \\
\hline Number of TooShort Reads & 30086 & 6 \\
\hline Number of Isogroups & 19522 & 13591 \\
\hline Avg Contig Count & 2.6 & 1.4 \\
\hline Number With One Contig & $11168 ; 57.2 \%$ & $11676 ; 85.9 \%$ \\
\hline Avg Isotig Count & 2.1 & 1.2 \\
\hline Number With One Isotig & 11213 & 11705 \\
\hline Number of Isotigs & 41407 & 16465 \\
\hline Avg Contig Count & 3.4 & 1.6 \\
\hline Largest Contig Count & 18 & 13 \\
\hline Number With One Contig & 11168 & 11676 \\
\hline Avg Isotig Size & 1514 & 705 \\
\hline N50 Isotig Size & 1905 & 839 \\
\hline Largest Isotig Size & 12895 & 3326 \\
\hline Number Of Large Contigs & 22001 & 7763 \\
\hline Avg Large Contig Size & 962 & 819 \\
\hline Number Of all Contigs & 51270 & 19120 \\
\hline
\end{tabular}

U. vulgaris and U. gibba transcriptomes assembled by Newbler 2.7.

significant BLAST hits, compared with the isotigs, may be due to their short sizes and also due to the presence of transcripts derived from microbes without any NCBI record.

The results of the GO annotations of the UT_U.gibba transcriptome are given in Table 2. The proportion of annotated isotigs is a bit lower and the proportion of

Table 2 GO Annotation summary

\begin{tabular}{llllll}
\hline & \multicolumn{2}{l}{ Isotigs } & & \multicolumn{2}{l}{ Singletons } \\
\cline { 2 - 3 } & U.vulgaris & U.gibba & & U.vulgaris & U.gibba \\
\hline NCBI nr & $96 \%$ & $89 \%$ & $40 \%$ & $47 \%$ \\
Number of GO terms & 212122 & 95741 & 90735 & 264218 \\
GO annotation & $73 \%$ & $75 \%$ & $25 \%$ & $39 \%$ \\
Biological process & $54 \%$ & $52 \%$ & $54 \%$ & $53 \%$ \\
Molecular function & $13 \%$ & $12 \%$ & $14 \%$ & $13 \%$ \\
Cellular component & $34 \%$ & $36 \%$ & $32 \%$ & $34 \%$ \\
E.C. assignment & $33 \%$ & $28 \%$ & & $25 \%$ & $33 \%$ \\
\hline
\end{tabular}

Values in \% indicate the percentage of sequences/groups with one or more significant blast hits/ annotations based on an e-value cut-off of $10^{-5}$. annotated singletons is a bit higher in $U$. gibba than in $U$. vulgaris. This difference results from a higher amount of unassembled reads in UT_U.gibba relative to UT_U. vulgaris. The proportion of isotigs with an assigned E.C. was also higher in $U$. vulgaris than in $U$. gibba.

Despite of the differences in cultivation conditions, plant tissues used for RNA extraction, cDNA library preparation and assembly parameters, the general partition of isotigs into basic KEGG categories was very similar between the two Utricularia species (Figure 1). "Catalytic activity" and "Binding" were the prevalent categories among Molecular Function. "Cell" and "Organelle" dominated in the Cellular Component section. Abundant categories "Metabolic Process" and "Cellular" were followed by slightly less numerous categories "Response to stimulus" and "Biological regulation". The high representation of the "Singleorganism process" category appeared due to co-existing microbes.

We summarized the results of our $U$. vulgaris transcriptome assembly and annotation and created a webaccessible database (http://utricularia.prf.jcu.cz/index.php) which can be easily searched by BLAST or annotation. 

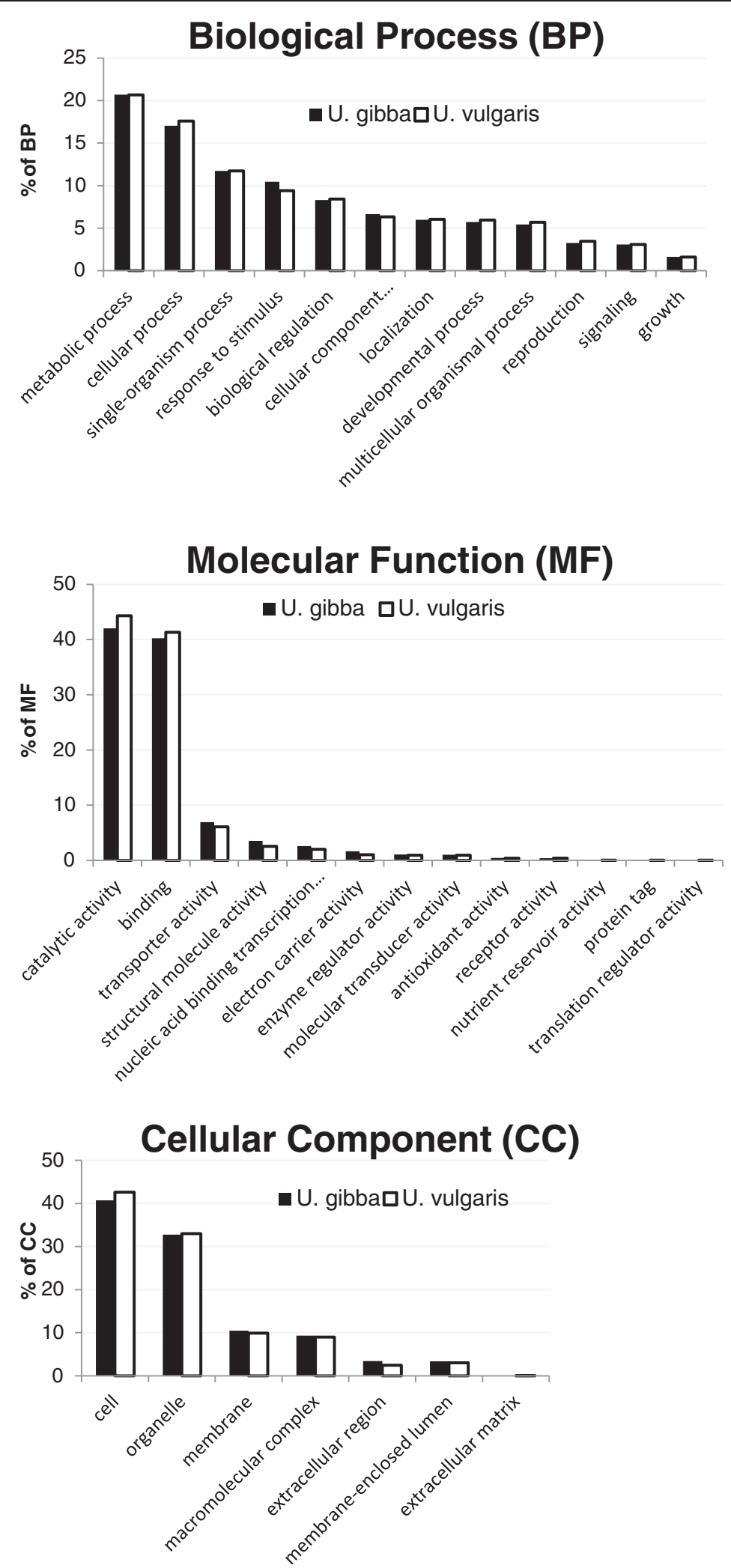

Figure 1 Distribution of GO categories. The comparison of the distribution of unique transcripts (isotigs and singletons) between $U$. gibba and U. vulgaris transcriptomes in three main $\mathrm{GO}$ categories. 


\section{The composition of transcriptomes}

More than $99 \%$ of the isotigs with significant hits were assigned by MEGAN to plants (Streptophytes) in both $U$. vulgaris and $U$. gibba. All remaining isotigs (38 in $U$. vulgaris and 87 in $U$. gibba) belonged to Fungi, Metazoa, unicellular eukaryotes, and prokaryotes (Figure 2). The taxonomic diversity of singletons was much higher: 5.3\% and $10.6 \%$ of singletons with significant hits were assigned outside the Streptophytes in $U$. vulgaris, and $U$. gibba, respectively (Additional file 1). The non-plant sequences were mostly derived from microbial commensals, as well as a minor fraction from animal (fish, worm) RNA contamination. The very low proportion of prokaryotic sequences was due to the polyA+ RNA used to prepare cDNA. As prokaryotic mRNAs rarely contain polyA+ tails, they were mostly eliminated. The proportion of non-plant transcripts is probably higher among singletons, because many of them may not have produced statistically significant hits due to incomplete microbial records in public databases. The abundance of microbederived transcripts was higher in the $U$. gibba transcriptome prepared only from plants grown under natural conditions and colonized with microbes. In contrast, the $U$. vulgaris transcriptome was constructed from a pooled RNA sample prepared from the plants cultivated under both sterile and non-sterile conditions.

\section{Large isogroups and alternative splicing}

The isogroups containing numerous isotigs may include transcripts derived from several or many loci, e.g. retroposons, or from transcripts undergoing AS [23]. The $U$. vulgaris transcriptome contained six isogroups with $>100$ isotigs, 23 isogroups with $>45$ isotigs, and 332 isogroups with $>10$ isotigs. The largest, isogroup 00018 in U.vulgaris, included 480 isotigs derived from various members of a large BETA GLUCOSIDASE gene family.

In contrast, the $U$. gibba assembly contained zero isogroups with $>100$ isotigs, only two isogroups with $>45$ isotigs, and 17 isogroups with $>10$ isotigs (Additional file 2). The main reason for such a high difference in the number of large isogroups with many isotigs between the UT_U. vulgaris and UT_U.gibba transcriptome assemblies seems to be the method of cDNA preparation. Normalization of the cDNA library led to the enrichment of rare transcripts in $U$. vulgaris, including alternatively spliced mRNAs. The largest isogroup in the UT_U.gibba assembly, which was generated without a cDNA normalization step, contained only 68 isotigs, representing transcripts coding for the small subunit of Rubisco, the most abundant protein on Earth. When read counts are extremely high, as in the case of Rubisco, sequencing errors occur in multiple reads which are then assembled into separate, artifactual contigs. Some large isogroups in $U$. gibba also represented transcripts derived from multiple loci-e.g. isogroup 00005 (KETOACYL COA SYNTHASE family) [24] or the isogroups 00002 and 00012, which gave no hits in BLAST searches of NCBI databases, but yielded multiple hits against the U. gibba genome draft (CoGe-id36222).

Alternative splicing appears to be the main reason for the transcript abundance and diversity in many of the

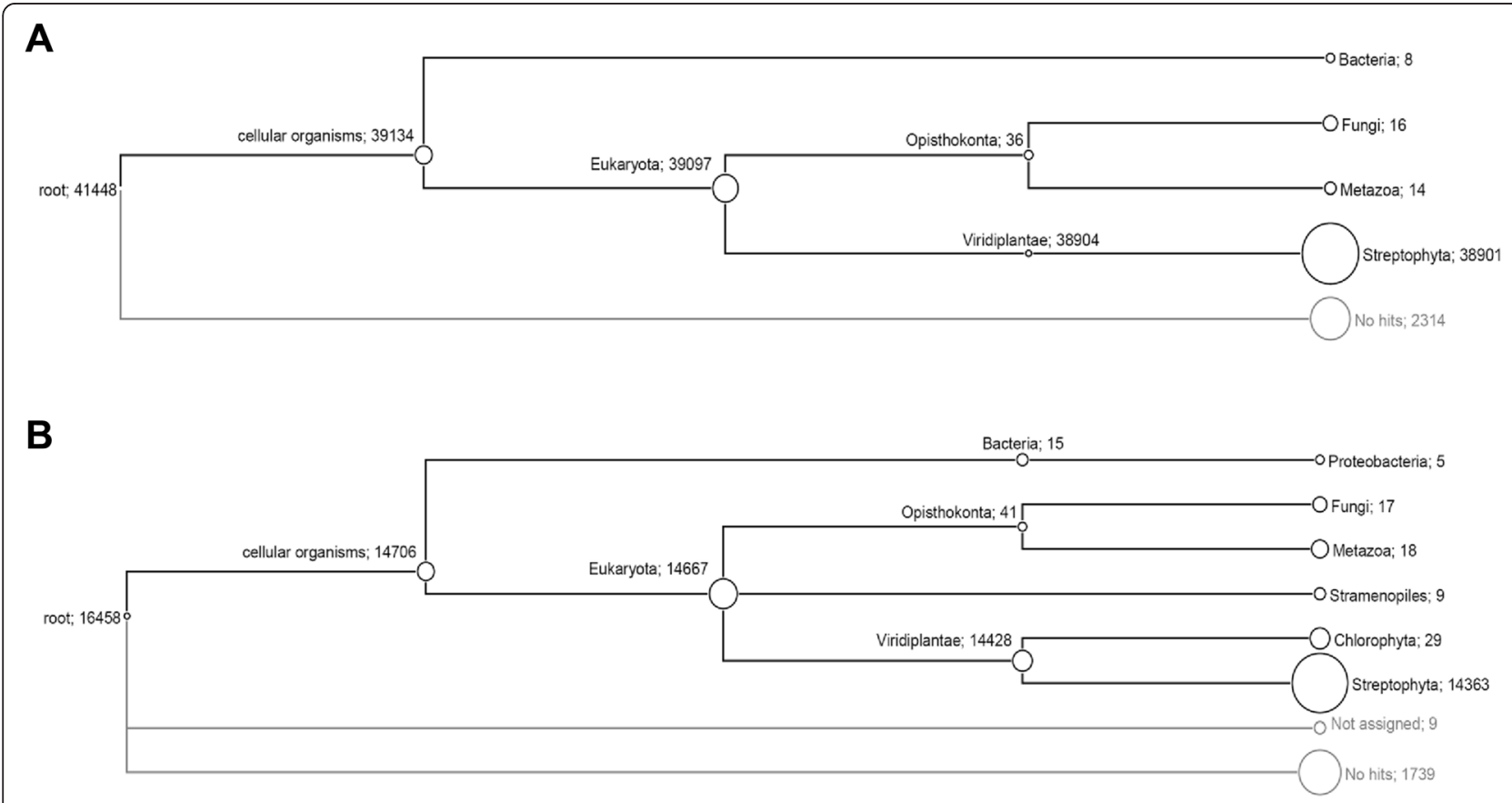

Figure 2 Taxonomic assignment. Dendrogram showing number of MEGAN assigned U. vulgaris (A) and U. gibba (B) isotigs. 
largest isogroups in $U$. vulgaris and in $U$. gibba. These isotigs contain contigs corresponding to Arabidopsis exons and also numerous contigs which may be assigned to introns based on their position between two exons. Additional file 2 compares the 23 and 17 largest isogroups of $U$. vulgaris and $U$. gibba, respectively. They represent various genes or gene families belonging to similar structure and function categories.

Only one large isogroup (00008) appears to be the same in both Utricularia species. It encodes a family of ATP dependent RNA helicases. Its Arabidopsis homologs (At5g11170, At5g11200) are involved in a wide range of RNA metabolism including pre-mRNA splicing, mRNA transport, turnover, translation initiation etc. [25,26]. They undergo AS, as documented by genome-wide analysis of transcript variants [27]. Five contigs of the isogroup 00008 in $U$. vulgaris match Arabidopsis exons, suggesting extensive AS of transcripts derived from at least two related genes. The more than four fold higher isotig count of the 00008 isogroup in $U$. vulgaris than in $U$. gibba may again reflect a significant enrichment in rare transcripts due to cDNA normalization of $U$. vulgaris transcriptome, or reflect a lower extent of AS in $U$. gibba. Three other isogroups of $U$. vulgaris could participate in the control of AS, including the isogroup 00013, encoding a homolog of $A F C 2$ protein kinase, which underwent extreme AS (producing multiple splice variants from the same primary transcript) in Arabidopsis [27]. The remaining large isogroups with AS code for membrane proteins with multiple domains, proteins involved in protein degradation, or fulfilling regulatory functions. Two large isogroups (00020, 00061) were assigned to retroposons in the $U$. vulgaris transcriptome. No large isogroup corresponding to transposons or retroposons was found in the $U$. gibba transcriptome, however three single isotigs were.

\section{Two isogroups with extreme alternative splicing}

We selected two isogroups of $U$. vulgaris with very high isotig counts for more detailed analysis. After aligning all 277 isotigs of the isogroup 00007, we found that all of them were derived from the same locus, because only one sequence variant (contig) corresponded to each exon of the homologous Arabidopsis gene, At1g27980, coding for sphingoid long-chain base 1-phosphate lyase (LCB-1-P lyase) (Additional file 3). We assigned eight contigs to eight introns based on a comparison with the homologous Arabidopsis gene. The retention of variable numbers of introns was responsible for the observed extreme AS in this isogroup. Only one isotig 00648 contained the correct ORF with genetic information for a functional protein. To confirm AS experimentally, we designed primers targeted to exon 6 or intron 6 (forward) and exon 15 (reverse) and ran PCR (Figure 3). The size of PCR fragment generated from genomic DNA (2.4 kb) with exon-specific primers

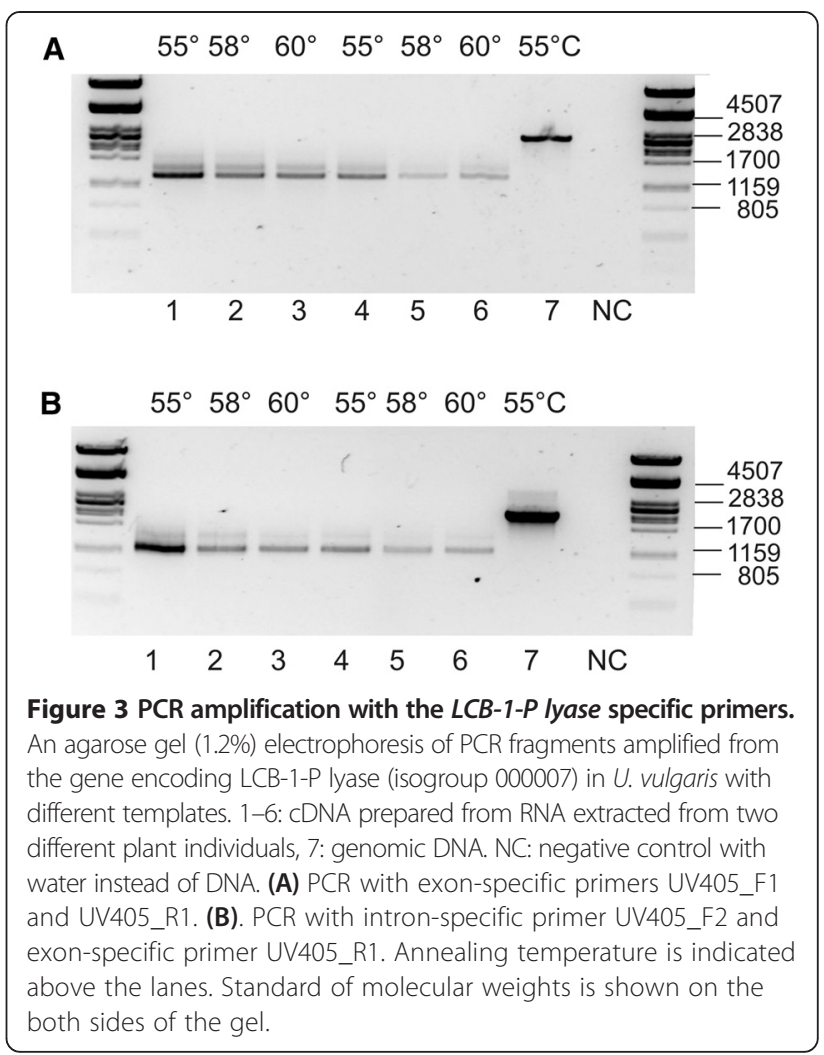

UV405_F1 and UV405_R1 agreed with the expected size of this genomic region (2,353 bp). The amplification of cDNA produced a strong band $(1.3 \mathrm{~kb})$ corresponding to correctly spliced mRNA with no introns (1,377 bp) and several weak upper bands most likely derived from partially spliced mRNA with retained introns. The primers spanning from intron 6 to exon 15 (UV405_F2 and UV405_R1) produced a PCR fragment from genomic DNA as well as one strong band $(1.1 \mathrm{~kb})$ and a few weaker ones from cDNA. The strong band amplified from cDNA provided evidence for intron 6 retention, because no amplification with this primer pair could occur if only correctly spliced mRNA were present in the transcript pool.

To achieve the correct assembly of alternative transcripts in a species without reference genome is very difficult. It becomes even more challenging if multiple similar paralogous genes are transcribed and alternatively spliced. In such cases, chimeric misassembled contigs are frequently generated [28]. The isogroup 00006 homologous to the ETHYLENE INSENSITIVE 2 EIN2 gene (At5g03280) in Arabidopsis is an example of the mixture of alternatively spliced transcripts derived from at least two loci. We identified contigs corresponding to the exons and introns of the EIN2 gene. Several putative exons existed in two sequence variants and occurred in chimeric isotigs. We confirmed the occurrence of two EIN2 gene copies by direct sequencing of $U$. vulgaris 
genomic DNA. We designed two primer pairs UV304_F1, R1 and UV308_F1, R1 (Additional file 4) and amplified and sequenced a part of exon 7 from both EIN2 paralogs. The alignment $(1,360 \mathrm{bp})$ of $U$. vulgaris sequences with EIN2 homologs across angiosperms was used in phylogenetic analysis to generate MP and ML trees (Figure 4). The trees constructed by both methods showed the same topology and confirmed a relatively recent duplication of EIN2, preceding the divergence of $U$. vulgaris and $U$. gibba. We found only one EIN2 homolog in the $U$. gibba genome (CoGe-id36222).

The ratio of non-synonymous and synonymous substitutions $(\mathrm{Ka} / \mathrm{Ks})$ in the pairwise comparison between EIN2-1 and EIN2-2 of $U$. vulgaris was 0.41 , between EIN2 of $U$. vulgaris and EIN2 of $U$. gibba was 0.44 (for both $U$. vulgaris EIN2 paralogs). The data suggest no variation in evolutionary constraints.

\section{Putative orthologs between $U$. vulgaris and $U$. gibba}

We performed a reciprocal BLAST hit search to identify putative orthologs between the UT_U.vulgaris transcriptome and a 19475-mRNA database derived from the genomic draft of $U$. gibba, which represents an in silico transcriptome of this species. We chose the $U$. gibba transcriptome derived from a genomic draft, because it supposedly represented more complete set of transcripts than the experimental transcriptome UT_U. gibba.

We identified 12,267 putative orthologous pairs, 10,600 of them contained $U$. vulgaris isotigs and the remaining 1,667 pairs contained $U$. vulgaris singletons. The orthologs represented about $42.9 \%$ of all genes annotated in the $U$. gibba genomic draft. We ordered putative orthologous pairs between $U$. gibba and $U$. vulgaris according to the sequence similarity of the regions aligned by BLAST. The distribution of orthologs assigned to individual similarity classes is shown in Figure 5. Most orthologous pairs

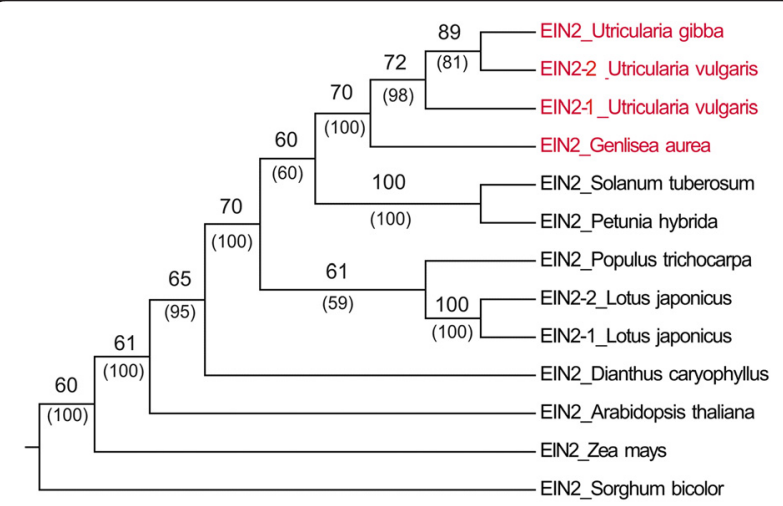

Figure 4 Phylogenetic analyses of EIN2. MP and ML tree constructed from the alignment of partial EIN2 sequences across angiosperms exhibited the same topology. Bootstrap supports calculated from 1000 pseudoreplicates are shown above branches (MP) or below branches in parentheses (ML).

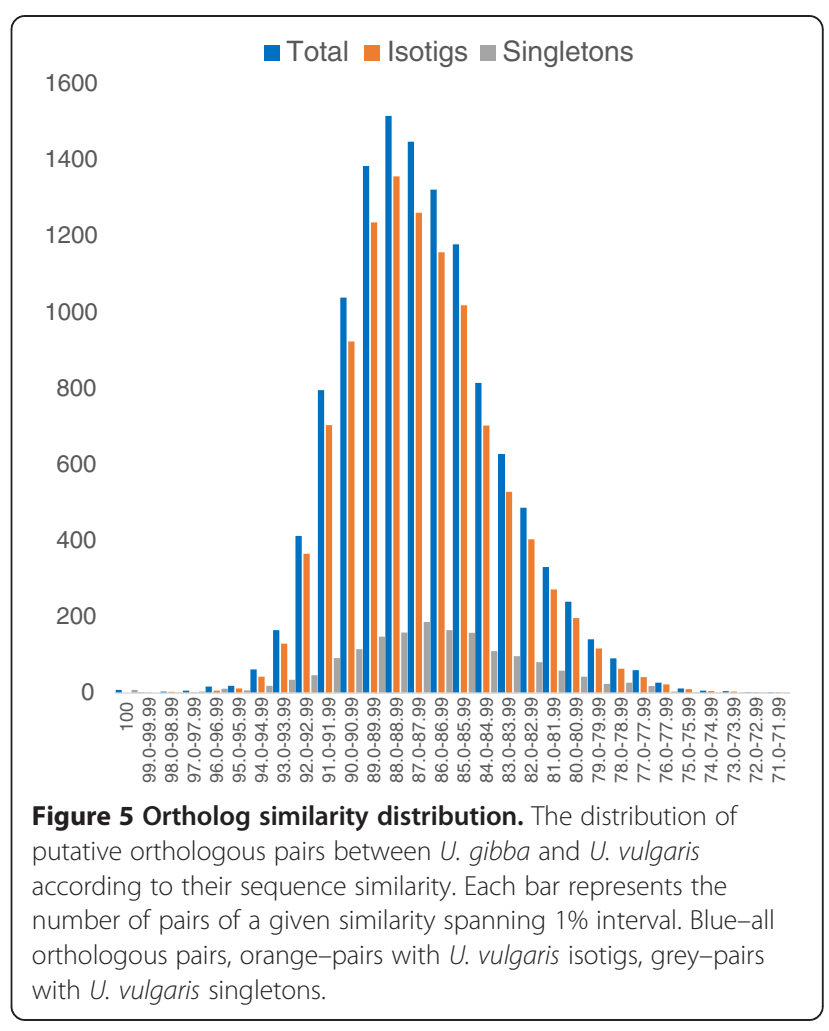

exhibited a sequence similarity of $85 \%-90 \%$, whether or not they included $U$. vulgaris isotigs or singletons. Singletons are much shorter than an average isotig (1,514 bp; Table 1), thus they often represent incomplete transcripts. Their sequence similarity depends on whether they are derived from a more or less conserved part of the gene, it does not reflect the similarity across an entire gene. For this reason, we performed the following analyses of the most conserved orthologs with the pairs containing only $U$. vulgaris isotigs, not singletons.

Because the overall sequence similarity of putative $U$. vulgaris- $U$. gibba orthologs was rather low (median 87\%), we investigated which GO categories were enriched among the most conserved orthologous pairs. GO enrichment (AgriGo) [29] analysis of the most conserved orthologs (with similarity higher than 93\%) against all orthologs identified 36 significantly enriched GO categories (Additional file 5). They belonged to the genes encoding proteins conserved across all angiosperms (ribosomal proteins, tubulins, small GTP-binding proteins, mitochondrial respiratory chain proteins, etc.). Their proportion in respective similarity classes of putative orthologs increased with increasing sequence similarity (Figure 6). Detailed inspection of GO categories enriched among highly conserved orthologs between $U$. gibba and $U$. vulgaris revealed genes which were less similar to their Arabidopsis counterparts than the rest of the highly conserved orthologs, namely MYOSIN XI B (homolog of At1g04160) and 


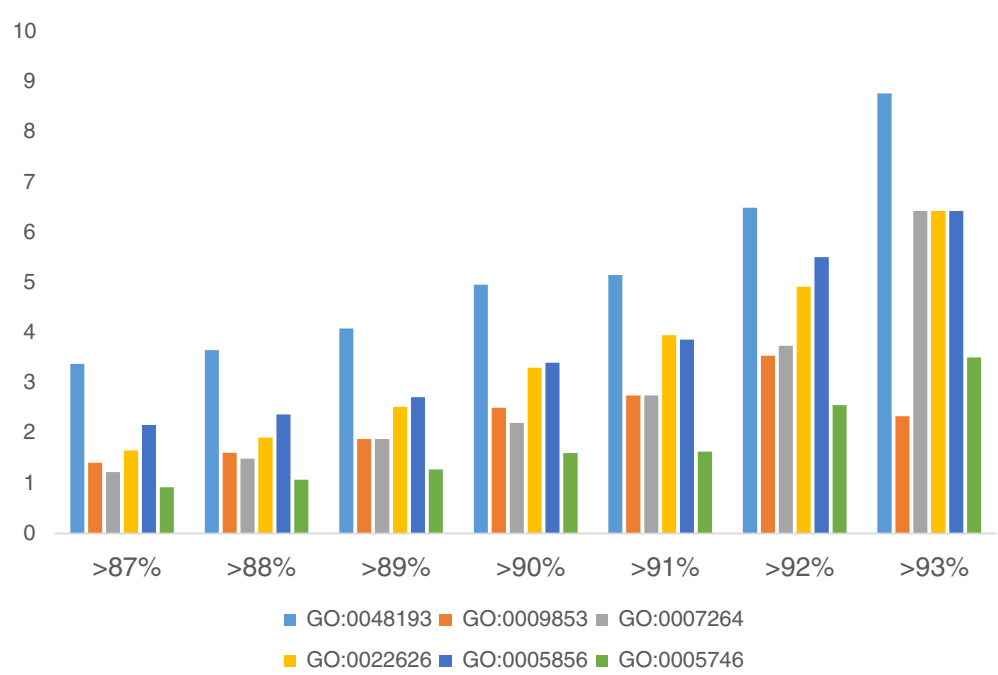

Figure 6 GO enrichment. The enrichment of particular GO categories (in \% of total GO categories) in the subsets of orthologous pairs between U. vulgaris and U. gibba with ascending sequence similarity. GO:0048193 Golgi vesicle transport; GO:0009853 Photorespiration GO: 0007264 Small GTPase mediated signal transduction; GO: 00022626 Cytosolic ribosomes; GO:0005856 Cytoskeleton; GO: 0005746 Mitochondrial respiratory chain.

TIP GROWTH DEFECTIVE 1 (TIP1) (a homolog of At5g20350). Interestingly, both genes play a role in root hair development in Arabidopsis. As neither $U$. gibba nor $U$. vulgaris produce roots, it is probable that the two genes have gained a novel or modified functions in Utricularia, explaining why their sequences are highly similar between both Utricularia species, but less similar to Arabidopsis homologs.

\section{Root-specific genes in rootless Utricularia}

As Utricularia vulgaris does not form roots, some of the genes involved in root development and function might have been lost. Ibarra-Laclette et al. [13] published a list of the genes associated with root in $A$. thaliana, but not found in the genome of $U$. gibba. They include MYB transcription factors, MADS box genes, cell-wall-associated kinases, nitrate transporter etc. (Ibarra Laclette et al. [13]). We selected the root-associated genes absent in $U$. gibba, supplemented additional genes exclusively or predominantly expressed in the roots of A. thaliana and checked their presence among putative orthologs identified by BLASTX-TBLASTN reciprocal BLAST search between $A$. thaliana protein data set (TAIR10_prot_20101214) and the UT_U.vulgaris transcriptome. We found a strong correspondence between the absence of particular genes in the $U$. gibba genome [13] and their absence in the $U$. vulgaris transcriptome (Additional file 6). Moreover, we did not find the counterparts of additional Arabidopsis root-associated genes in the $U$. vulgaris transcriptome, notably transcription factors involved in root hair (e.g. ROOT HAIR DEFECTIVE 6-RHD6, WEREWOLF-WER) or root cap development (e.g. BEARSKIN 1-BRN1, FEZ, SOMBRERO-SMB). These genes were also missing in the $U$. gibba genome (CoGeid36222). On the other hand, some genes such as AUXIN RESPONSE FACTOR 4 (ARF4) or INDOLE-3-ACETIC $A C I D$ INDUCIBLE (IAA) genes found putative orthologs in both the $U$. vulgaris transcriptome and the $U$. gibba genome (Additional file 6). Six of $13 \mathrm{U}$. vulgaris rootassociated genes (isotigs or single reads) under study contained complete ORFs, the rest of isotigs represented partial sequences, which reflected an overall incompleteness of experimental transcriptomes.

Because the transcriptomes are incomplete, the absence of an ortholog in the transcriptome, by itself, is not the evidence of its absence in the corresponding genome. Thus, we may not exclude the possibility that the absence of any respective gene in the $U$. vulgaris transcriptome was caused by its low or missing transcription. However, the coincident absence of 48 root-associated genes and concordant presence of 11 root-associated genes in the $U$. vulgaris transcriptome and the $U$. gibba genome suggest that, at least in the case of root genes, the UT_U. vulgaris transcriptome reflects the gene content of the $U$. vulgaris genome.

Interestingly, two copies of the gene AUXIN RESPONSE FACTOR 16 (ARF16), duplicated in the U. gibba genome [13], were also found in the $U$. vulgaris transcriptome. The full agreement between the sets of root-associated genes lost in $U$. gibba and $U$. vulgaris and the concordance of the genes duplicated in both species support the notion that deletions and duplications of genes involved in root-associated genes occurred before the divergence of the two Utricularia species. 


\section{Discussion}

\section{Transcriptome comparison}

Recent progress in next generation sequencing makes it possible to sequence the genomes and transcriptomes of non-model plants to an unprecedented extent. The 1000 plants (one KP or 1KP) initiative (https://sites.google.com/ a/ualberta.ca/onekp) is just one example of current efforts. The genomic draft of $U$. gibba [13] has attracted attention because it represents one of the smallest genomes in the plant kingdom and opened the possibility to study the mechanisms responsible for genome contraction in plants. The availability of a sequenced genome and an experimental transcriptome of $U$. gibba generated by 454 pyrosequencing from various organs [12], made $U$. gibba a suitable species for comparative transcriptomics in Utricularia. We chose a temperate congener $U$. vulgaris as a counterpart for comparison. Both species share an aquatic carnivorous life style, lack of roots, and display rapid apical shoot growth, but exhibit partly distinct ecophysiology (turion formation in $U$. vulgaris, possible terrestrial life in $U$. gibba; see [1].

We utilized the two kinds of transcriptomes available for $U$. gibba in our comparative studies. The in silico transcriptome derived from the genomic draft is assumed to be more complete than the experimental transcriptome, which is known to lack transcripts due to low or missing gene expression [21]. However, an in silico transcriptome is only as good and complete as the annotation of the genome of interest. It may also erroneously assign virtual transcript to a pseudogene which is not expressed. In contrast, an experimental transcriptome is comprised of real transcripts able to capture alternatively spliced transcripts derived from the same gene. Considering advantages and disadvantages of the two kinds of transcriptomes, we decided to prefer the in silico transcriptome for the identification of putative orthologs between $U$. gibba and $U$. vulgaris. In contrast, experimental transcriptome of U. gibba [12] was used to compare expressed gene categories and also to identify alternatively spliced transcripts.

The transcriptome of $U$. gibba [12] was prepared from inflorescences in addition to submersed parts of plants, but, unlike $U$. vulgaris transcriptome, it did not include sterile plants. Another distinction was the application of cDNA normalization to the construction of the $U$. vulgaris transcriptome but not the $U$. gibba transcriptome. Despite these differences, the proportions of annotated GO categories were very similar (Figure 1).

The distribution of GO categories in Utricularia was also in line with previously published transcriptomic data from carnivorous species of Sarracenia [30]. This study used only a quarter of the data of the $U$. vulgaris transcriptome, without pooling various tissues or developmental stages. Despite methodological differences, the proportions of GO categories were very similar between
$U$. vulgaris and Sarracenia. Only the categories "Response to stimulus" and "Biological regulation" were much higher in Utricularia than recorded for Sarracenia. This difference may reflect distinct life styles of both carnivorous genera. Whereas Sarracenia is a robust slowly growing terrestrial perennial plant, Utricularia is a fast growing aquatic plant which has to cope with sudden changes of environment (nutrient level, salinity, streaming or even temporary desiccation). Alternatively, the impact of very different data sets cannot be excluded. In this case, it would affect only the two GO categories, which appears unlikely.

\section{Examples of alternative splicing}

Normalization of CDNA is recommended for the study of AS, because it increases the proportion of rare mRNAs, often represented by alternatively spliced transcripts. This approach revealed that $61.2 \%$ of intron-containing genes were alternatively spliced in Arabidopsis [27]. We cannot directly compare the extent of AS in $U$. vulgaris and Arabidopsis, because missing genomic information in $U$. vulgaris prevents the accurate assignment of splice variants. However, 23 largest isogroups in $U$. vulgaris (Additional file 2) matched Arabidopsis homologs with more than 3 splice variants belonging to $25 \%$ of the genes with the highest level of AS in Arabidopsis [27], which suggests that a similar set of genes is highly alternatively spliced in $U$. vulgaris and Arabidopsis.

The gene encoding ATP dependent RNA helicase (isogroup 00008) was shown to be alternatively spliced not only in $U$. vulgaris, but also in $U$. gibba. It participates in the control of mRNA splicing and export in Arabidopsis $[25,26]$ and its expression is regulated by AS in this plant. It is therefore possible that the paralogs encoding ATP dependent RNA helicase (isogroup 00008) play similar roles in Utricularia.

We also documented extreme AS in the isogroup 00007 in $U$. vulgaris homologous to the gene for LCB-1-P lyase in Arabidopsis. The function of LCB-1-P lyase or sphingosine-1-phosphate (SPH-1-P) lyase in plants is not fully understood. Ng et al. [31], Coursol S et al. [32] described the role of SPH-1-P as a lipid messenger in guard cell abscisic acid (ABA) response. Nishikawa et al. [33] showed that LCB-1-P was degraded by LCB-1-P lyase (encoded by AtDPL1, At1g27980), which was located in the endoplasmic reticulum. LCB-1-P lyase regulates LCB-1-P content and through this activity participates in stomata closure and dehydration stress response in Arabidopsis [33,34].

Our $U$. vulgaris transcriptome was generated from submersed plant organs which did not develop stomata. However, we have verified that above-water flower stems of $U$. vulgaris contain stomata (Adamec, unpublished results). It is therefore possible that transcripts with retained 
introns represent a pool from which functional transcripts may be readily formed by additional splicing when LCB-1-P lyase becomes necessary. This protein may be needed when the submersed plant body continues to grow above water. A similar regulatory role of intron retention was observed, for example, in the fern Marsilea vestita, where transcripts with retained introns were stored in spores and spliced after germination [35].

The expression of the gene for LCB-1-P lyase in submersed plant organs lacking stomata may also suggest that it fulfills a distinct function in Utricularia, not associated with stomata. As SPH-1-P affects ion channels in guard cell protoplasts [32], we speculate that this lipid messenger may have a role in water pumping regulation in Utricularia traps, which is associated with potassium channels in trap bifid glands [36]. Finally, it is possible that extreme AS in the isogroup 00007 coding for LCB-1-P lyase does not have any regulatory function and represents an error in a complex splicing process. To our knowledge, AS of transcripts encoding LCB-1-P lyase has not been studied in any plant species. We cannot determine whether this also occurs in $U$. gibba, because nonnormalized transcriptomes have only limited potential to detect AS.

\section{Duplication of the EIN2 gene in $U$. vulgaris}

We found a duplication of EIN2 gene in $U$. vulgaris transcriptome. This gene is essential for ethylene signaling and occurs in a single copy in many plant species and its duplication is rare among angiosperms [37,38]. Two EIN2 paralogs undergoing accelerated evolution were recently identified in Lotus japonicus [39]. They regulate not only a response to ethylene, but also nodulation in the course of symbiosis with rhizobia. The two EIN2 paralogs in Utricularia vulgaris may be important for the interaction between plants and microbes, similar to the role of the two EIN2 genes in the symbiosis between leguminous plants and bacteria [39]. We speculate that the duplication of the EIN2 gene occurred early in the evolution of the genus Utricularia and might have been associated with the transition to a carnivorous life-style. Subsequently, one copy was lost in $U$. gibba. Similar $\mathrm{Ka} / \mathrm{Ks}$ ratios in pairwise comparisons of Utricularia EIN2 genes do not indicate any shift in function. However, it should be emphasized, that only parts of the $U$. vulgaris EIN2 genes, confirmed by Sanger sequencing, were analyzed. The examination of additional species of Lentibulariaceae regarding EIN2 multiplication will shed light on the evolution and function of this important gene.

\section{Low sequence similarity between putative U. gibba-U. vulgaris orthologs}

The median value of sequence similarity among 12,267 putative orthologous pairs (measured as high-scoring segment pair of BLAST alignments) between the two Utricularia species was only $87 \%$ (Figure 5), much lower than for example a median ortholog similarity between two Corylus species (98\%)- [40] or between chimpanzee and human (about 93.7\%- [41]) which belong to different genera. $U$. vulgaris and $U$. gibba are classified in the same generic section Utricularia [1,5], although they are not sister species. The reason for the high divergence between $U$. gibba and $U$. vulgaris appears to be a high substitution rate, described in Lentibulariaceae as one of the characteristics of the plant carnivorous syndrome [11,42]. However, these two species still displayed very high sequence similarity in orthologs encoding ribosomal proteins, components of respiratory chain and cytoskeletal proteins, which were ultraconserved across the plant kingdom. We also observed a high conservation of some genes involved in root-associated function, which were generally less conserved among angiosperms. This could be explained by a functional shift shared by the two rootless aquatic Utricularia species.

\section{The loss of root-associated genes}

We found a perfect coincidence between the absence of root-associated genes in the $U$. gibba genome [13] and the absence of their counterparts in the $U$. vulgaris transcriptome. The correspondence between both Utricularia species was also observed in additional root-associated genes, not specifically analyzed by [13] (Additional file 6). Although the absence of particular genes in our transcriptome may be due to intrinsic incompleteness of any experimental transcriptomic data, the high coincidence between genomic and transcriptomic gene occurrences in two species suggests that many root-associated genes are indeed missing in the $U$. vulgaris genome. It is probable that the loss of root-associated genes had occurred already in the ancestor of $U$. gibba and $U$. vulgaris. The comparison of the presence or absence of root-associated genes in additional Utricularia species will be very useful for understanding the adaptation to an aquatic rootless carnivorous life-style.

Besides gene losses, gene duplications could be also very informative regarding the evolution and consequences of aquatic carnivory in plants. For example, the duplication of ARF16 in $U$. gibba [13] was also observed in the $U$. vulgaris transcriptome. In contrast, the EIN2 duplication event was unique for $U$. vulgaris.

\section{Conclusions}

Our study is the first example of comparative transcriptomics in the species-rich genus Utricularia. We compared the transcriptome of $U$. vulgaris with the previously published transcriptome of $U$. gibba [12] and confirmed a general similarity of their expression profiles. Both Utricularia species displayed some distinctions from 
the Sarracenia transcriptome [30] due to the aquatic life-style different from a terrestrial carnivory. Average sequence similarity of the putative orthologs between $U$. vulgaris and $U$. gibba was lower than $90 \%$, which could be caused by elevated substitution rate in this genus [11]. A strong correspondence in presence or absence of root-associated genes between $U$. vulgaris and $U$. gibba suggests that the loss of some root-specific genes had occurred before the two rootless species separated. Future transcriptome and genome comparisons over numerous Utricularia species will certainly deepen our understanding of plant evolution and adaptation.

\section{Methods}

\section{Plant material}

We utilized $U$. vulgaris from three sources for RNA extraction: (1) sterile plants from a meristem tissue culture (courtesy of Ing. Kamil Pásek, Ostrava-Poruba, Czech Republic), growing in a half-strength Gamborg B5 liquid medium with $500 \mathrm{mg} \mathrm{l}^{-1} \mathrm{KNO}_{3}$, microelements, vitamins, and $2.5 \%$ sucrose, but without other organic substances [43]; (2) sterile plants transplanted axenically from tissue culture into an aerated mineral medium [44], with reduced amounts of phosphorus and nitrogen where plants were grown in $16 / 8 \mathrm{~h}$ light/dark regime of fluorescent light at $23^{\circ} \mathrm{C}$ for 4 weeks, attaining the length of approximately $20 \mathrm{~cm}$; and (3) plants with colonized traps, approximately $35 \mathrm{~cm}$ in length, cultivated in a 750 litre outdoor plastic container closely simulating natural dystrophic conditions [45], with the litter of robust sedges (Carex spp.) used as a substrate [45] and partial shading (usually $20-50 \%$ of incident irradiance). Plants cultivated outdoor were occasionally fed fine zooplankton prey to support growth. No experiments on humans or animals, or cloning experiments were carried out. All activity complied with ethics rules.

Tissue for RNA extraction was harvested in the middle of the dark period to reduce the amount of Rubiscocoding mRNA in samples, frozen immediately in liquid nitrogen, and stored at $-70^{\circ} \mathrm{C}$ until further processing.

\section{RNA extraction, cDNA library construction, and 454 sequencing}

Total RNA was extracted from entire shoots, including leaves and traps, from all three sources described above. Inflorescences were not used in this study. Approximately $800 \mathrm{mg}$ of fresh weight (2 individuals) of whole shoots in three replicates from each source were ground in liquid nitrogen using a mortar and pestle and RNA was extracted using RNeasy Plant Midi Kit (Qiagen Inc., Valencia, CA, USA). The results of the parallel extractions were pooled in equal proportions into a single sample and the quality and quantity of the extracted RNA were verified by gel electrophoresis and by spectrophotometer, measuring the 230/260 ratio with Biophotometer (Eppendorf, Germany).

A pooled RNA sample was provided to GATC Biotech (Konstanz, Germany) for the construction of a cDNA library and subsequent sequencing. Library construction involved DNAselection for polyadenylated (polyA+) transcripts to enrich for protein-coding mRNAs, DNase I treatment and normalization through denaturation/ reassociation of $\mathrm{cDNA}$, to improve the representation of low-copy transcripts and thereby maximize gene discovery. The resulting library was sequenced with a full picotiter plate on a 454 GS-FLX sequencer with Titanium reagents (Roche Applied Science, Indianapolis, IN, USA), using standard 454 protocols.

\section{Transcriptome assembly and annotation}

Raw reads obtained by 454 pyrosequencing were submitted to European Nucleotide Archive under the study accession number PRJEB8057 (http://www.ebi.ac.uk/ena/data/ view/PRJEB8057).

We assembled our raw reads with Newbler 2.7 using the following command line settings:-cdna,-it 500,-ig 1000,-icc 200. The subsequent annotation steps excluded singletons that were $<100 \mathrm{bp}$ in length. Annotation of isotigs (contigs assembled by Newbler and roughly corresponding to individual transcripts) and singletons (transcripts represented by single reads) was done using the BLAST2GO pipeline [46] with the following parameters: Annotation rule cutoff 55 , e-value $10^{-6}$, Hit-HSP overlap 0, GO weight 5.

MEGAN v 5.7.0 [47] with default parameters was used to get the taxonomical annotation of isotigs and singletons. MEGAN uses BLASTX output and LCA (Lowest Common Ancestor) classifier based on the actual NCBI taxonomy to obtain the taxonomical annotations.

\section{Search for orthologs}

The orthologous transcripts between $U$. vulgaris and $U$. gibba were identified by means of reciprocal BLAST search [48]. We used the transcriptome of $U$. vulgaris containing both isotigs and the singletons which passed quality filtering (min phred score 25, min length 100, max ambiguities $0, \max$ homopolymer 8 ). As the $U$. gibba transcriptome published by Ibarra-Laclette et al. [12] might have been incomplete, we downloaded the database 19475-mRNA, constructed from the complete genome of $U$. gibba, from CoGe website (https://genomevolution. org/CoGe/-id36222). We generated local databases and used BLASTN, threshold e-10, to retrieve the best hits in reciprocal BLAST searches. Reciprocal BLAST outputs were compared by $\mathrm{RBH}$ (reciprocal blast hit) script and putative orthologs which appeared as the same pair in both BLAST outputs were identified. The similarity of 
orthologs in \% was based on high-scoring segment pair (HSP) of BLAST alignments.

The orthologs between U.vulgaris and Arabidopsis (TAIR10_prot_20101214) were searched in a similar way, with BLASTX (threshold e-10) and TBLASTN (threshold e-10) instead of BLASTN, which means that predicted protein sequences were aligned.

\section{The analysis of large isogroups}

We selected the isogroups containing more than 45 isotigs in $U$. vulgaris and more than 10 isotigs in $U$. gibba and found their Arabidopsis counterparts using the BLASTX search in TAIR (www.arabidopsis.org). We assigned their functions based on TAIR annotations. We aligned all isotigs from two selected isogroups of $U$. vulgaris, each displaying an extremely high number of isotigs, by MUSCLE implemented in Geneious 7.1.5. We identified introns retained in the transcripts with the help of Layout output from Newbler 2.7 and by comparison with gene structures in Arabidopsis (TAIR).

\section{GO enrichment analysis}

We performed the analysis of enrichment of particular GO categories among putative $U$. gibba-U. vulgaris orthologs by means of AgriGO (http://bioinfo.cau.edu.cn/agriGO) [29]. We input the list of annotated putative orthologs as a customized annotated reference and the list of orthologs with specific similarity as a customized annotation. We applied Yekutieli multi-test adjustment, significance level 0.05 and a minimum number of mapping entries of 4 .

\section{RT PCR confirmation}

To verify transcript sequences inferred from the 454 assembly, we collected $U$. vulgaris plants grown outdoor and isolated total RNA by RNeasy Plant Mini Kit (Qiagen). RNA was treated with DNase I (DNA-free, Ambion, TX, USA) to eliminate a DNA contamination. The treatment was repeated until no amplification was observed in PCR reaction with RNA as a template. One $\mu \mathrm{g}$ of RNA and oligo dT primers (500 ng) were heated $5 \mathrm{~min}$ at $65^{\circ} \mathrm{C}$, chilled on ice and mixed with Transcriptor buffer (Roche Scientific, Mannheim, Germany), $0.5 \mu$ of Protector RNase Inhibitor (Roche Scientific), $2 \mu \mathrm{l}$ of $10 \mathrm{mM}$ dNTPs and 10 units of Transcriptor Reverse Transcriptase (Roche Scientific). The first strand of cDNA was synthesized at $55^{\circ} \mathrm{C}$ for $30 \mathrm{~min}$ and then amplified with the primers (Additional file 4) targeted to isotig 00405 (isogroup 00007). PCR was run in a T Gradient cycler (Biometra, Goettingen, Germany) with Taq polymerase (Promega, Madison, WI, USA) under the following conditions: initial denaturation 2 min at $94^{\circ} \mathrm{C}$; 36 cycles consisting of $40 \mathrm{~s}$ at $93^{\circ} \mathrm{C}, 45 \mathrm{~s}$ at given annealing temperature and $2 \mathrm{~min}$ at $72^{\circ} \mathrm{C}$; final extension $10 \mathrm{~min}$ at $72^{\circ} \mathrm{C}$.

\section{PCR confirmation}

Genomic DNA from $U$. vulgaris plants grown outdoor was isolated by the sorbitol extraction [49]. PCR with the primers (Additional file 4) targeted to isotigs 00304 or 00306 (isogroup 00006) was run as described above at annealing temperature $60^{\circ} \mathrm{C}$. PCR products were Sanger sequenced and partial genomic sequences corresponding to two paralogs of EIN2 in U. vulgaris were deposited in GenBank under the numbers KP279653 and KP279654.

\section{Phylogenetic analyses}

We downloaded EIN2 sequences of $\mathcal{U}$. gibba (Sc00341 g15851 from CoGe-id36222), Genlisea aurea (EPS70151), Solanum tuberosum (XM006354273), Petunia hybrida (AY353249), Populus trichocarpa (XM002326149), Lotus japonicus (chr5.CM1729.230 and chr1. CM0012.1100), Dianthus caryophyllus (HQ441183), Arabidopsis thaliana (NM12040), Zea mays (AY359584), and Sorghum bicolor (XM002457067). We aligned these angiosperm EIN2 sequences with partial protein sequences EIN2-1 and EIN22 from $U$. vulgaris and created a 1,361 amino acid long gapped-alignment in Geneious 7.1.5. We then adopted a maximum parsimony (MP) approach with stepwise addition to the starting tree, no topology constraints, tree bisectionreconnection (TBR) swap, and 1000 bootstrap pseudoreplicates to construct a 60\% consensus tree in PAUP"4 v10 [50]. Gaps were treated as missing characters. To confirm phylogenetic positions of the EIN2 paralogs in Utricularia we performed Maximum Likelihood (ML) analysis in PAUP $* 4$ v10 [50] of the same nucleotide alignment under HKY85 evolution model, using the same tree construction methods as described above, 1000 bootstrap pseudoreplicates.

\section{Availability of supporting data}

We summarized the results of our $U$. vulgaris transcriptome assembly and annotation and created a web-accessible database (http://utricularia.prf.jcu.cz/index.php) which can be easily searched by BLAST or annotation. Raw reads obtained by 454 pyrosequencing were submitted to European Nucleotide Archive under the study accession number PRJEB8057 (http://www.ebi.ac.uk/ena/data/view/ PRJEB8057). The sequences of EIN2 paralogs were deposited in GenBank under the numbers KP279653 and KP279654. The data sets undelying phylogenetic EIN2 studies was submitted to TreeBase under the ID 17158. The sequences of $U$. gibba were obtained from the study of Ibarra-Laclette et al. [12] (doi:10.1186/1471-2229-11-101).

\section{Additional files}

Additional file 1: Dendogram showing number of MEGAN assigned $U$. vulgaris $(\mathrm{A})$ and $U$. gibba (B) singletons. 


\section{Additional file 2: Isogroups with numerous isotigs in $U$. vulgaris} and U. gibba.

Additional file 3: Layout of the isotigs comprising the isogroup 00007. Exons are marked in yellow, contigs corresponding to exons and introns are blue.

Additional file 4: PCR primers used in this study.

Additional file 5: GO categories significantly enriched among $U$. gibba-U. vulgaris putative orthologs with sequence similarity higher than $93 \%$.

Additional file 6: Root-associated genes in U. vulgaris and U. gibba.

\section{Abbreviations}

ARF: AUXIN RESPONSE FACTOR; AS: Alternative splicing; BRN: BEARSKIN DDBJ: DNA Data Bank of Japan; EIN: ETHYLENE INSENSITIVE GO, Gene Onthology; HSP: high scoring segment pair; IAA: INDOLE-3-ACETIC ACID INDUCIBLE; LCB-1-P: long-chain base 1-phosphate; MP: maximum parsimony; RBH: reciprocal blast hit; RHD: ROOT HAIR DEFECTIVE; TBR: tree bisectionreconnection; SBM: SOMBRERO; SPH-1-P: sphingosine-1-phosphate; TIP: TIP GROWTH DEFECTIVE; WER: WEREWOLF.

\section{Competing interests}

The authors declare that they have no competing interests.

\section{Authors' contributions}

JB extracted RNA, performed transcriptome assembly and annotation, and contributed to manuscript preparation. JDS and MAC provided troubleshooting and advice with sequence analyses and contributed to manuscript preparation JP wrote custom scripts and provided computer assistance. DS contributed to experimental design, performed plant cultivation, collected plant material, and contributed to manuscript preparation. LA contributed to experimental design, optimized the composition of cultivation media, and contributed to manuscript preparation. HS optimized RNA extraction, designed data analyses, performed phylogenetic analysis, GO enrichment and AS analysis, set up a major part of the manuscript. All authors read and approved the final manuscript.

\section{Acknowledgements}

The authors are grateful to Jaroslav Vrba and Jakub Borovec for their valuable contribution to plant cultivation, and to Daniel B Sloan for advice on web site construction and transcriptome assembly. They are also thankful to Naoki Takebayashi for bioinformatics advice, Kamil Pásek for providing axenic tissue cultures of U. vulgaris, and Kateřina Haškovcová for excellent technical assistance. Access to computing and storage facilities owned by parties and projects contributing to the National Grid Infrastructure MetaCentrum, provided under the programme 'Projects of Large Infrastructure for Research is greatly acknowledged. This work was funded by the grant of the Grant Agency of the Czech Republic P504/11/0783 to LA, and further supported by Czech Research Programme No. RVO67985939 to LA, and by the project "Integration of the experimental and population biology using new methods of interdisciplinary issues-the way to excellence with young scientists," Reg.No.: CZ.1.07/2.3.00/30.0048, funded by the European Social Fund (ESF) and the state budget of Czech Republic through the Operational Program Education for Competitiveness (OPEC)" to JDS and HS.

\section{Author details}

'Faculty of Science, University of South Bohemia, Branišovská 31, České Budějovice 37005, Czech Republic. ${ }^{2}$ Institute of Experimental Botany CAS, Rozvojová 263 6- Lysolaje, Praha 16502, Czech Republic. ${ }^{3}$ Institute of Botany CAS, Zámek 1, Průhonice 25243, Czech Republic. ${ }^{4}$ Institute of Botany CAS, Section of Plant Ecology, Dukelská 135, Treboň 37982, Czech Republic. ${ }^{5}$ Institute of Fundamental Sciences, Massey University, Private Bag 11222, Palmerston North 4442, New Zealand.

Received: 22 December 2014 Accepted: 23 February 2015

Published online: 07 March 2015

\section{References}

1. Taylor P. The Genus Utricularia: A Taxonomic Monograph. London: Kew Bulletin; 1989. Additional Series XIV.
2. Guisande C, Granado-Lorencio C, Andrade-Sossa C, Duque SR. Bladderworts. Funct Plant Sci Biotechnol. 2007;1:58-68.

3. Adamec L. Ecophysiological look at plant carnivory: Why are plants carnivorous? In: Seckbach J, Dubinski Z, editors. All Flesh is Grass. Plant-Animal Interrelationships. Cellular Origin, Life in Extreme Habitats and Astrobiology. Volume 16. Dordrecht - Heidelberg - London - New York: Springer Science + Business Media B. V; 2011. p. 455-89.

4. Greilhuber J, Borsch T, Müller K, Worberg A, Porembski S, Barthlott W. Smallest angiosperm genomes found in Lentibulariaceae, with chromosomes of bacterial size. Plant Biol. 2006:8:770-7.

5. Veleba A, Bureš $P$, Adamec L, Šmarda P, Lipnerová I, Horová L. Genome size and genomic GC content evolution in the miniature genome-sized family Lentibulariaceae. New Phytol. 2014;203:22-8.

6. Jobson RW, Albert VA. Molecular rates parallel diversification contrasts between carnivorous plant sister lineages. Cladistics. 2002;18:127-36.

7. Müller K, Borsch T, Legendre L, Porembski S, Theisen I, Barthlott W. Evolution of carnivory in Lentibulariaceae and the Lamiales. Plant Biol. 2004;6:477-90.

8. Müller K, Borsch T, Legendre L, Porembski S, Barthlott W. Recent progress in understanding the evolution of carnivorous Lentibulariaceae (Lamiales). Plant Biol. 2006;8:748-57.

9. Müller K, Borsch T. Phylogenetics of Utricularia (Lentibulariaceae) and molecular evolution of the trnK intron in a lineage with high substitutional rates. Plant Syst Evol. 2005;250:39-67.

10. Albert VA, Jobson RW, Michael TP, Taylor DJ. The carnivorous bladderwort (Utricularia, Lentibulariaceae): a system inflates. J Exp Bot. 2010;61:5-9.

11. Ibarra-Laclette E, Albert VA, Herrera-Estrella A, Herrera-Estrella L. Is GC bias in the nuclear genome of the carnivorous plant Utricularia driven by ROS-based mutation and biased gene conversion? Plant Signal Behav. 2011;6:1631-4.

12. Ibarra-Laclette E, Albert VA, Perez-Torres CA, Zamudio-Hernandez F, de Ortega-Estrada MJ, Herrera-Estrella A, et al. Transcriptomics and molecular evolutionary rate analysis of the bladderwort (Utricularia), a carnivorous plant with a minimal genome. BMC Plant Biol. 2011;11:101.

13. Ibarra-Laclette $E$, Lyons $E$, Hernández-Guzmán G, Pérez-Torres CA, Carretero-Paulet L, Chang TH, et al. Architecture and evolution of a minute plant genome. Nature. 2013;498:94-8.

14. Peroutka M, Adlassnig W, Volgger M, Lendl T, Url WG, Lichtscheidl IK Utricularia: a vegetarian carnivorous plant? Algae as prey of bladderwort in oligotrophic bogs. Plant Ecol. 2008;199:153-62.

15. Sirová D, Borovec J, Černá B, Rejmánková E, Adamec L, Vrba J. Microbial community development in the traps of aquatic Utricularia species. Aquat Bot. 2009;90:129-36.

16. Sirová D, Borovec J, Šantrůčková H, Šantrůček J, Vrba J, Adamec L. Utricularia carnivory revisited: Plants supply photosynthetic carbon to traps. J Exp Bot. 2010;61:99-103.

17. Sirová D, Borovec J, Picek T, Adamec L, Nedbalová L, Vrba J. Ecological implications of organic carbon dynamics in the traps of aquatic carnivorous Utricularia plants. Funct Plant Biol. 2011;38:583-93.

18. Sirová D, Šantrůček J, Adamec L, Bárta J, Borovec J, Pech J, et al. Dinitrogen fixation associated with shoots of aquatic carnivorous plants: is it ecologically important? Ann Bot. 2014;114:125-33.

19. Adamec L. Different reutilization of mineral nutrients in senescent leaves of aquatic and terrestrial carnivorous Utricularia species. Aquat Bot. 2014;119:1-6.

20. Vincent $O$, Weisskopf $C$, Poppinga S, Masselter T, Speck T, Joyeux M, et al. Ultra-fast underwater suction traps. Proc R Soc B. 2011;278:2909-14.

21. Martin JA, Wang Z. Next-generation transcriptome assembly. Nat Rev Genet. 2011;12:671-82.

22. Ge XX, Chen HW, Wang HL, Shi AP, Liu KF. De novo assembly and annotation of Salvia splendens transcriptome using the Illumina platform. PLoS One. 2014;9:e87693.

23. Reddy ASN, Marquez Y, Kalyna M, Barta A. Complexity of the alternative splicing landscape in plants. Plant Cell. 2013;25:3657-83.

24. Joubes J, Raffaele S, Bourdenx B, Garcia C, Laroche-Traineau J, Moreau P, et al. The VLCFA elongase gene family in Arabidopsis thaliana: phylogenetic analysis, 3D modelling and expression profiling. Plant Mol Biol. 2008:67:547-66.

25. Kammel C, Thomaier M, Sorensen BB, Schubert T, Laengst G, Grasser M, et al. Arabidopsis DEAD-Box RNA Helicase UAP56 interacts with both RNA and DNA as well as with mRNA export factors. PLoS One. 2013;8:e60644.

26. Owttrim GW. RNA helicases and abiotic stress. Nucl Acids Res. 2006;34:3220-30.

27. Marquez Y, Brown JWS, Simpson C, Barta A, Kalyna M. Transcriptome survey reveals increased complexity of the alternative splicing landscape in Arabidopsis. Genome Res. 2012;22:1184-95. 
28. Yang Y, Smith S. Optimizing de novo assembly of short-read RNA-seq data for phylogenomics. BMC Genomics. 2013;14:328.

29. Du Z, Zhou X, Ling Y, Zhang Z, Su Z. agriGO: a GO analysis toolkit for the agricultural community. Nucl Acids Res. 2010;38:W64-70.

30. Srivastava A, Rogers WL, Breton CM, Cai L, Malmberg RL. Transcriptome analysis of Sarracenia, an insectivorous plant. DNA Res. 2011;18:253-61.

31. Ng CKY, Carr K, McAinsh MR, Powell B, Hetherington AM. Drought-induced guard cell signal transduction involves sphingosine-1-phosphate. Nature. 2001;410:596-9.

32. Coursol S, Fan LM, Le Stunff H, Spiegel S, Gilroy S, Assmann SM. Sphingolipid signalling in Arabidopsis guard cells involves heterotrimeric G proteins. Nature. 2003;423:651-4.

33. Nishikawa M, Hosokawa K, Ishiguro M, Minamioka H, Tamura K, Hara-Nishimura I, et al. Degradation of sphingoid long-chain base 1-phosphates (LCB-1Ps): Functional characterization and expression of AtDPL1 encoding LCB-1P lyase involved in the dehydration stress response in Arabidopsis. Plant Cell Physiol. 2008:49:1758-63.

34. Nakagawa N, Kato M, Takahashi Y, Shimazaki K, Tamura K, Tokuji Y, et al. Degradation of long-chain base 1-phosphate (LCBP) in Arabidopsis: functional characterization of LCBP phosphatase involved in the dehydration stress response. J Plant Res. 2012;125:439-49.

35. Boothby TC, Zipper RS, van der Weele CM, Wolniak SM. Removal of retained introns regulates translation in the rapidly developing gametophyte of Marsilea vestita. Dev Cell. 2013;24:517-29.

36. Sydenham PH, Findlay GP. The rapid movement of the bladder of Utricularia sp. Aust J Biol Sci. 1972;26:1115-26.

37. Alonso JM, Hirayama T, Roman G, Nourizadeh S, Ecker JR. EIN2, a bifunctional transducer of ethylene and stress responses in Arabidopsis. Science. 1999;284:2148-52.

38. Shibuya K, Barry KG, Ciardi JA, Loucas HM, Underwood BA, Nourizadeh S, et al. The central role of PhEIN2 in ethylene responses throughout plant development in petunia. Plant Phys. 2004;136:2900-12.

39. Miyata K, Kawaguchi M, Nakagawa T. Two distinct EIN2 genes cooperatively regulate ethylene signaling in Lotus japonicas. Plant Cell Phys. 2013;54:1469-77.

40. Ma H, Lu ZQ, Liu BB, Qiu Q, Liu JQ. Transcriptome analyses of a Chinese hazelnut species Corylus mandshurica. BMC Plant Biol. 2013;13:152.

41. Nielsen R, Bustamante C, Clark AG, Glanowski S, Sackton TB, Hubisz MJ, et al. A scan for positively selected genes in the genomes of humans and chimpanzees. PLoS Biol. 2005;3:e170.

42. Wicke SK, Schaeferhof B, DePamphilis CW, Muller KF. Disproportional plastome-wide increase of substitution rates and relaxed purifying selection in genes of carnivorous Lentibulariaceae. Mol Biol Evol. 2014;31:529-45.

43. Adamec L, Pásek K. Photosynthetic $\mathrm{CO}_{2}$ affinity of aquatic carnivorous plants growing under nearly-natural conditions and in vitro. Carniv Plant Newslett (Fullerton). 2009;38:107-13.

44. Sato C, Schnoor JL, McDonald DB, Huey J. Test medium for the growth of Nitrosomonas europaea. Appl Environ Microbiol. 1985;49:1101-7.

45. Adamec L. How to grow Aldrovanda vesiculosa outdoors. Carniv Plant Newslett (Fullerton). 1997;26:85-8.

46. Conesa A, Gotz S, Garcia-Gomez JM, Terol J, Talon M, Robles M. BLAST2GO: a universal tool for annotation, visualization and analysis in functional genomics research. Bioinformatics. 2005;21:3674-6.

47. Huson DH, Auch AF, Qi J, Schuster SC. MEGAN analysis of metagenomic data. Genome Res. 2007;17:377-86.

48. Gabaldon T, Koonin EV. Functional and evolutionary implications of gene orthology. Nat Rev Genet. 2013;14:360-6.

49. Štorchová H, Hrdličková R, Chrtek Jr J, Tetera M, Fitze D, Fehrer J. An improved method of DNA isolation from plants collected in the field and conserved in saturated $\mathrm{NaCl} / \mathrm{CTAB}$ solution. Taxon. 2000;49:79-84.

50. Swofford DL. PAUP*: Phylogenetic analysis using parsimony (*and other methods). Version 4. Sunderland, Massachusetts: Sinauer Associates; 2003.

\section{Submit your next manuscript to BioMed Central and take full advantage of:}

- Convenient online submission

- Thorough peer review

- No space constraints or color figure charges

- Immediate publication on acceptance

- Inclusion in PubMed, CAS, Scopus and Google Scholar

- Research which is freely available for redistribution

Submit your manuscript at www.biomedcentral.com/submit 